\title{
The use of email as a component of adult stammering
}

\section{therapy: a preliminary report}

Carolyn R. Allen, Speech and Language Therapy, University of Strathclyde, Glasgow, UK

Contact address: $\quad$ Speech and Language Therapy Division, Sir Henry Wood Building, Jordanhill Campus, Southbrae Drive, Glasgow, G13 1PP

Email:

carolyn.allen@strath.ac.uk

Declarations: $\quad$ There are no declarations of funding to make and there are no competing interests. 


\section{Summary}

In West Glasgow email has evolved from being a quick means of arranging therapy appointments with adults who stammer into an exchange of therapeutic messages for some clients. Drawing on therapist experience and existing online therapy and telehealth literature, this paper presents the benefits of integrating technology into usually technology-free clinical practice, which include improving access to services, supporting speech change, facilitating lasting personal growth, improving clinical decision making, equalising the therapist-client relationship and enhancing caseload management. The effectiveness and ethics of, and the rationale for, clinical practice that includes email use need to be considered and are discussed. Excerpts from emails exchanged with three clients are used to support the argument that email can appropriately complement face-to-face therapy. It is recognised that research is required to formally evaluate the client experience.

\section{Introduction}

Stammering, also known as stuttering or dysfluency, is defined as ${ }^{1}$ 'overt speech symptoms will include some or all of the following: part word repetitions, prolongations, and/or blocking. Coping strategies, for example substituting difficult words, situation avoidance, or changes in nonverbal behaviour may occur early or develop over time. In addition, fear of stammering may cause psychological or emotional distress' (p.73). 
In the United Kingdom speech and language therapy (SLT) intervention with adults who stammer may address the overt dysfluencies alone or a combination of speech behaviours, the related negative emotions and life impact, with the choice of therapy approach being influenced by both the therapist's evaluation and the client's reported concerns. Therapy is instructional, in teaching specific speech management techniques, and supportive, using counselling skills to support a client's changing relationship with their stammering. Clients usually attend for face-to-face therapy appointments, initially on a weekly basis with less frequent contacts during periods of consolidation of new communication skills.

The use of telehealth in SLT continues to emerge worldwide. Recent reviews that have considered adult communication disorders reveal that most SLT-related telehealth comprises the use of videoconferencing in the management of neurological communication disorders, ${ }^{2,3}$ although this is also being used in postlaryngectomy care ${ }^{4}$ and voice therapy. ${ }^{5}$ In specific relation to adult stammering, videoconferencing has been used in Canada with one adult client to avoid lengthy travel for clinic-based follow-up support after an intensive course. ${ }^{6}$ The therapist was able to provide feedback regarding most speech behaviours and the client was able to use the alternative therapy medium to help consolidate his new communication skills across different speaking situations. In Australia, a randomised control trial has compared face-to-face and telehealth delivery of an existing fluency-focussed treatment programme. ${ }^{7}$ Twenty telehealth participants received therapy instruction, feedback and support through a combination of telephone and email contact and twenty face-to-face participants attended for therapy in a clinic setting. Immediate and 12-month post-treatment reduction in stammering was achieved equally by both groups, with greater efficiency reported for the telehealth group who required fewer 
contact hours on average to achieve equitable gains. With regards the use of email in adult stammering there is currently only one descriptive report of therapeutic emails being exchanged between an American therapist and a dysfluent adult in Bosnia-Herzegovina every two or three months over a three year period. The client described the informal exchanges as self-therapy which supported him in successfully developing problem solving skills. ${ }^{8}$

It is recognised that there are opportunities to incorporate technology more widely in stammering treatment. ${ }^{9}$ Using email in Glasgow began as a creative response to difficulties arranging face-to-face sessions when clients' work or study commitments prevented in-clinic attendance, but is becoming a clinical tool in its own right. However, the glamour and novelty of a new means of service delivery are not sufficient justifications for its use and therefore this paper aims to address the question: is it appropriate to consider integrating email communication in to stammering therapy?

\section{Method}

Since 2004 , sixteen clients have used email to communicate with the author as part of their therapy programme. Client ages range from 19 to 52 years and with the exception of two clients, all have been male. Severity of overt and covert stammering both ranged from mild to severe. Therapeutic intervention was based on individual presentation, blending speech modification techniques and counselling support in both face-to-face appointments and email exchanges. Email was used in response to client need, broadly serving two functions; administrative and therapeutic. Of the 
472 emails exchanged across the group, 328 (69.5\%) were primarily administrative, in arranging face-to-face appointments. The other 144 emails (30.5\%) were primarily therapeutic, in monitoring ongoing treatment goals or offering problem-solving guidance. Often email messages contained both administrative and therapeutic elements. Figure 1 reflects this diverse combination of email types and face-to-face (FTF) intervention for the sixteen clients, presented in order of referral from 20042009.

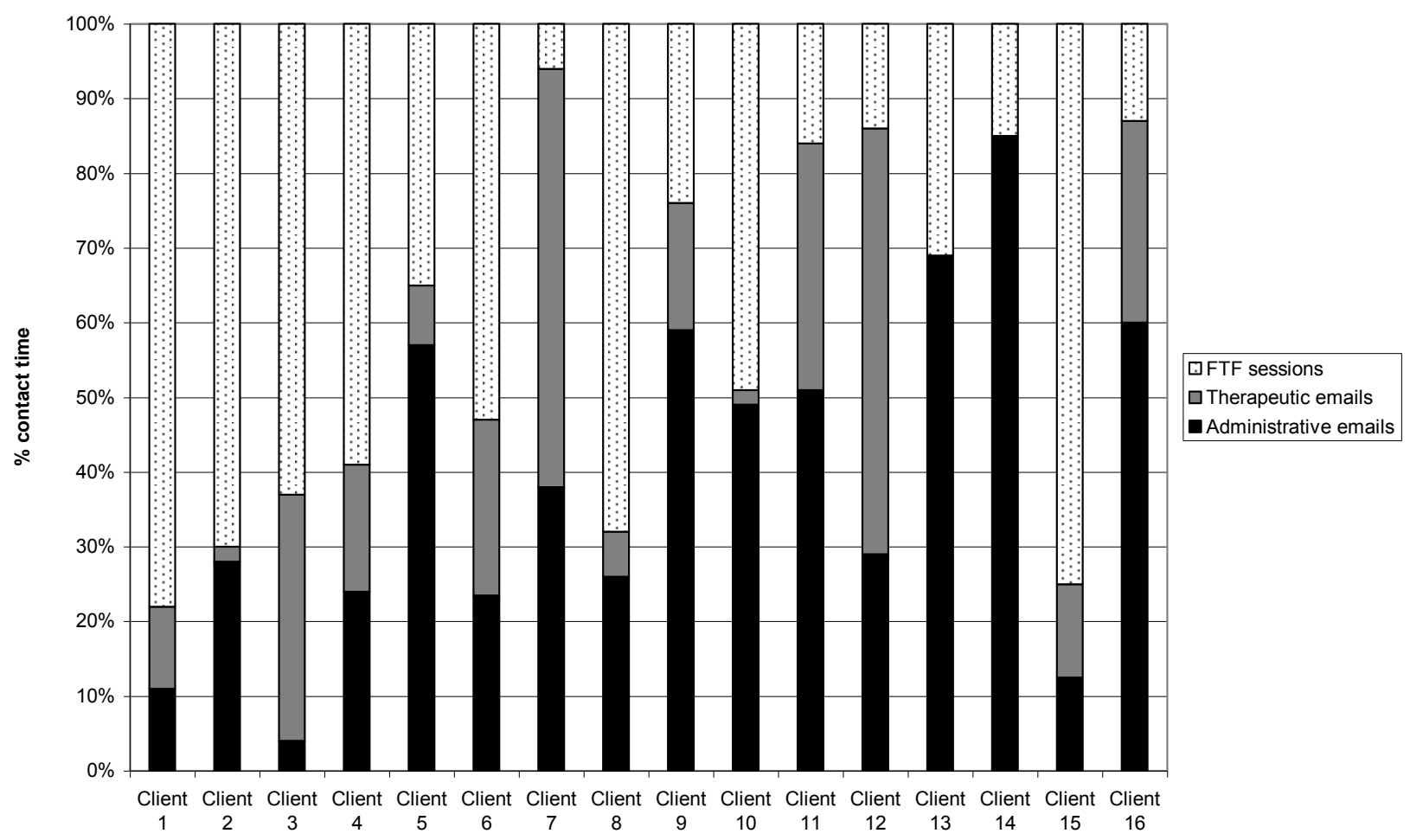

Figure 1. Percentage spread of contact type across face-to-face intervention, therapeutic emails and administrative emails.

\section{Results}


Of the sixteen clients who have used emails as part of therapy, eleven have been discharged, two of whom moved out of the area and one failed to respond to email, letter or telephone correspondence. The remaining eight were discharged having satisfactorily reduced dysfluent speech symptoms, avoidance behaviours and the negative impact of stammering as recorded by pre- and post-therapy scores on the Wright \& Ayre Stuttering Self Profile ${ }^{10}$ and through therapist-client discussion. With therapy content being driven by individual needs in a clinical, not research, setting a meaningful inter-client comparison of the number of face-to-face sessions and emails exchanged with related treatment outcomes is not possible. The following discussion therefore draws on past clinical use, the therapist experience and the existing literature to present the potential advantages for using email with an adult stammering caseload.

\section{Discussion}

\section{Easier access to therapy services}

In response to a perceived stigma surrounding stammering, some adults who stammer (AWS) do not wish to admit they stammer. If they do not wish to acknowledge stammering to their General Practitioners in order to gain a referral to

SLT, and are additionally fearful of using the telephone, ${ }^{11}$ email may be a less threatening medium through which contact with the SLT service can be made.

Access to services through the use of telehealth has been recognised as potentially quicker ${ }^{12}$ and email-initiated referrals do remove the time required for a General Practitioner (GP) to dictate and post a referral letter. A shortened waiting time may 
be important for some clients who could be feeling unsettled by the prospect of change and the unknown nature of the therapy ahead of them. The email extract below demonstrates this potential degree of urgency for one prospective client. He received a response within 24 hours, a much shorter time from his decision to enquire about therapy than if he had needed to approach his GP to request a referral to SLT:

"Please see below my email to BSA Scotland and their reply with a reference to yourself. I thought I should strike while the iron is hot and contact you."

\section{An alternative means of communication}

Difficulties in producing speech may result in partial conversations due to the premature ending of these through frustration or the time limits of a session. For reasons that go beyond communication impairment, writing may be easier than talking and therefore emailing the therapist in addition to face-to-face appointments could offer some communicative relief in being able to express concerns, ask questions or offer reflections more easily than if using the spoken word.

\section{Transfer and maintenance of speech modification techniques}

Speech techniques initially learnt in the quiet, supportive clinic environment can be difficult to transfer immediately and independently into every day conversation with its increased pace and demand on conversational skills. Email allows for clarification of technique instructions and problem-solving between appointments, with clients 
able to re-read therapist guidance as many times as they wish. ${ }^{13,14}$ An example of therapist advice is below:

"when you are using the technique, remember to pause after the difficult word, making sure this is a constructive pause in which you think about how to make the sound / word easier. After the pause say the word again.....and then you can continue the rest of the sentences. If you're not completely sure what you're doing I can clarify further."

Any client using email will be accessing their account in a setting other than the clinic. Taking the experience of therapy in to a more natural environment through telehealth is thought to increase the client's use of techniques in everyday conversations. ${ }^{15}$ 'Checking-in' with the therapist regularly to record that practice is being completed may additionally support client commitment to practice:

"I felt my problem has not got any better. It is most essential that I must play my part in the game now."

\section{Supporting self-reflection}

Through their written reflections, clients can experience an event and related emotions again in the fullest sense. Choosing the appropriate words to describe the experience can facilitate greater understanding of a situation, ${ }^{16}$ as does externalising the problem so that, in this case, stammering can be seen from a more detached 
perspective. ${ }^{13}$ New self-knowledge gained from written reflections may be surprising, but valuable, as this sample suggests:

"Funny, I started off this email with the intention of breaking ties with you and I realise that it is helpful for me to say what I have just said in the paragraph above which I wouldn't have done if I hadn't emailed you."

The asynchronous nature of email can further be supportive in offering the client a longer opportunity for reflection than would occur in a synchronous face-to-face session. This time lag can be similarly advantageous for a therapist in having additional time to construct a deeply informative response to the client. ${ }^{17}$

\section{Privacy}

It has been recognised within online counselling that greater self-disclosure may occur when there is no risk of a visible negative listener reaction, or when clients wish to express strong emotions, such as crying, privately. ${ }^{18}$ With masculinity defined in part by 'emotional stoicism'19 this visual anonymity may well be an advantage for the predominantly male dysfluency caseload. However, complete anonymity is not appropriate for clients already feeling isolated ${ }^{18}$ and this is one reason that clients in this service are not permitted to receive therapy exclusively via email.

\section{Maintaining an equal therapist-client relationship}


Some clients consider the therapist to be in a position of authority, a standing often automatically granted to health professionals perceived by clients as having greater knowledge than themselves. Heightened sensitivity to their communication difficulties in possible contrast with the fluency of the therapist may also distract some clients from fully engaging with therapy. Whether the client perceives an imbalance between therapist and client authority from differences in fluency skills or knowledge, the less personal means of communicating via email may foster a more equal relationship, ${ }^{20,21}$ encouraging clients to share their thoughts more freely.

\section{Informed clinical decision making}

Although decisions regarding therapy goals are made jointly by the client and therapist, information about the lived experience of stammering is required before the therapist is able to suggest a plan. For clients who struggle to communicate verbally in the clinic, the amount of information available to the therapist can be compromised. With fluent dialogue via email, the therapist may gain access to supplementary, useful information allowing early clinical decision making and prompt therapeutic support.

Email allows clients to share their automatic response to a situation. The first of the two excerpts below evidences acute client concern prior to a presentation. The second excerpt describes the quite different response to the actual situation. Had each situation been described retrospectively at a therapy appointment, the description of events surrounding the presentation may have been less rich. 
"Now I found out that next Wednesday, we have an advocacy class. . .the difference between Wednesday's informal presentation and next week is that, next week there can be no word substituting. . . I am considering taking next Wednesday off, it is really hurting because I have had to pay xx for this course."

[the next therapy session provided guidance regarding the management of speaking anxieties]

"Actually I did end up going to the class. All week, I told myself I was not going to go, but the turning point was when our tutor told us that some fear the class so much that they drop out of the course.... I had to attend the class - for my own sake, otherwise my self-esteem would have taken a battering. I had to take a gamble that day. . I I remembered the written advice you had given me regarding presentations to settle my breathing, and to take my time, start things slowly, and walk up to the podium very slowly. . . to put it short - your advice was brilliant - it worked like nothing ever has before."

\section{A service that reaches beyond the clinic and clinic hours}

Distance may be a barrier to accessing SLT services, and although telehealth in Scotland has a focus on increasing service access for those disadvantaged by remoteness, telehealth is also beneficial when used locally. ${ }^{12}$ Return travel from educational, work or home base to the clinic can become inconvenient for regular attendance. ${ }^{15}$ Using email allows AWS to continue to access therapy services but without excessive disruption to their day, and as many email accounts can be 
accessed worldwide, clients can also remain in contact whilst working or travelling abroad $^{14}$ as this example demonstrates:

"I am now in Norway. I am still working on my problem but feel confident that I have seen the light at the end of the tunnel."

Therapeutic writing with a pen and paper has been suggested as being like "having a private therapist day and night"16 and the all-hours access to email similarly allows clients to engage with their virtual therapy world at a time that accommodates other commitments and allows them to share their thoughts when inspired..$^{13}$

\section{Promoting self-managed care}

The UK professional body for speech and language therapists recommends that clients are monitored at 3, 6 and 12 months and 2 years post-therapy to ensure therapy is beneficial in the long-term. ${ }^{1}$ Within these periods, clients will rely far less on their clinician for direct feedback and more on their own self-evaluation. Email permits infrequent contact between therapist and client, encouraging clients to become skilled at developing their own therapy plans and taking responsibility for their own change, thought to come from the active client engagement required in email use. $^{14}$

This excerpt from a longer email detailing the difficulties a client was experiencing in maintaining a focus on speech management is evidence of them using their reflections to plan their own therapy: 
"Anyway, I am glad I have written this because I will now revisit the John Harrison writings and see how/if I can mould the thinking to my present position. I don't think I need to see you at the moment UNLESS you are picking up anything from this email that makes you think it would be good to meet again - it's your call. But I really do appreciate the contact with you - it's good to know you are there if things really do go to pieces for some reason - but why should they? (positive energy!!)"

\section{Caseload management}

Using email to communicate with patients has raised concerns regarding an increased workload ${ }^{22}$ although these have been refuted elsewhere. ${ }^{23,24} \mathrm{~A}$ significant increase in workload through the addition of therapeutic emails has not emerged for the author; in fact, rather than creating an unmanageable workload, it is considered to augment caseload management as the time taken to send an email reply is less than the time that would be required for the equivalent in-clinic session. Waiting time for therapy for new clients may be reduced if the need for in-clinic appointments for current clients is reduced. ${ }^{21}$ It is recognised, however, that with increased numbers of clients communicating via email, it may be necessary in the future to allocate dedicated time within each working day for email responses.

\section{Considerations when incorporating email in to stammering therapy}

Whilst arguments have been presented for incorporating email in to adult stammering therapy, it is not the author's intention to suggest that therapy can solely be provided by correspondence. This departure from reliance on face-to-face 
therapy needs careful consideration before suggesting this to a client as a clinical option.

\section{Ethical practice}

The risk of interception when the internally secure NHS server connects with an external server is considered small but it is widely recognised that clients need to be made aware of all risks relating to privacy and confidentiality in telehealth. ${ }^{25,26}$ Clients are informed of the possible benefits and security risks in using email and email their consent in advance of exchanging ex-correspondence. Although this does not increase the security of email, this is currently considered best practice by the IT policy of the author's NHS Board and the standards of conduct, performance and ethics of the UK Health Professions Council (HPC). Readers working under different country law or clinical guidelines require to seek local advice regarding ethical constraints, before exchanging emails with clients.

Case records are a necessary part of health interventions as evidence of client contact and management plans but unlike written records composed from the therapist's recall and understanding of the session, the printouts of emails sent and received offer an exact record of the therapeutic exchange. These could be argued to be a more trustworthy account of events should this ever need scrutinised in the future, ${ }^{13}$ given that the permanent record 'forces impeccable ethics' (p. 27).

\section{Therapist-client relationship}


Online therapy challenges three of the expected features of therapy, in that it is usually face-to-face, relies on talking to communicate and occurs in real time. ${ }^{20}$ It is therefore unsurprising to read of scepticism regarding the ability to form a strong, intimate therapeutic relationship. ${ }^{27}$ However, more recent studies suggest that it is still possible to establish a strong working alliance even when not face-to-face, ${ }^{7,17,28}$ at times facilitating greater therapist confidence and positivity around their therapeutic relationships. ${ }^{28}$ Certainly it is the author's belief that some email exchanges have allowed for deeper insight into client experiences than face-to-face conversations alone, maintaining, if not enriching, the existing therapeutic relationship. The words in therapeutic emails have been described as being 'alive and fresh' (p.101) ${ }^{17}$ and having access to clients' lived experiences of stammering through detailed and honest accounts of events outside of the clinic should be considered a therapeutic privilege.

\section{Misunderstandings of written communication}

Just as an email printout can be beneficial in being an exact record of a therapeutic conversation, that same email may hold unintended messages at either first glance without full attention being paid to the message, or when the email is read repeatedly and the phrases studied take on new meanings. ${ }^{29}$ As no client has exclusive access to email therapy, face-to-face contact allows for resolution of any misunderstandings but therapists using email do need to be comfortable with silences between emails and the risk of misreading. More explicitly worded emails may compensate for potential issues regarding a lack of perceived emotion or erroneous interpretations of the message. ${ }^{13,17}$ 


\section{Lack of response to therapist-initiated emails}

An existing challenge is the technological equivalent of the client who fails to attend. Although an appointment has not been allocated to the client, there is uncertainty as to whether the client has forgotten to check their emails, been too busy to check, is no longer interested in therapy or whether technological difficulties prevent email access. Currently a second email is sent including the original email and if no response occurs this is followed up with a written letter asking the client to inform the department of their intentions.

\section{Clinician acceptance}

For email therapy to be introduced as an additional therapeutic option for dysfluent clients a therapist needs to be comfortable with IT. ${ }^{14,30}$ It has been emphasised previously that the introduction of technology is not intended to fully replace face-toface therapy and for clinicians hesitant of using a computer in a people-centred profession, reassurance may be taken from recognition that clinician input does not become redundant, it simply changes in its form. ${ }^{15}$

\section{Unproven effectiveness}

With a heavy emphasis in the UK health service on evidence-based practice (EBP), a paucity of efficacy or effectiveness studies regarding the integration of email into 
therapy leaves this practice open to criticism. However, if EBP is considered as a dynamic, continually evolving process that responds to client need by combining research evidence, client story and personal experience,${ }^{31} \mathrm{EBP}$ is being incorporated in to therapy; email is responding to client demands within and external to clinic sessions whilst following the same therapeutic principles as face-to-face therapy. Careful consideration regarding email is given before suggesting this to any client, practice adheres to the HPC practice standards and no client is permitted exclusive access to e-therapy, retaining the safety mechanism of traditional face-to-face service delivery.

\section{Reimbursement}

To date emails have only been exchanged with clients living, working or studying within the catchment area of the author's employing Health Board. Should our understanding of the role and effectiveness of email intervention increase it may be possible to provide therapy more remotely, offering rural and isolated clients access to specialist services. Reimbursement issues would need to be addressed for crossHealth Board service provision.

This paper has been written from a therapist's perspective; research with a focus on the client perspective is now clearly needed. This should include consideration of the experience of receiving therapy through a non face-to-face method in addition to evaluating the gains made in reducing stammering and the associated negative 
emotions or impact and the strength of email in helping clients to maintain new communication skills.

\section{Conclusion}

Goss \& Anthony have encouraged psychotherapists to involve themselves in the use of technology as a therapeutic medium, ${ }^{29}$ emphasising that to not do so would 'risk doing our clients a disservice' (p.225). Has the time come for speech and language therapists to subscribe to this belief too?

This paper has presented the novel use of email in response to the needs of one client group, drawing on therapist experiences, client emails and the existing literature regarding online therapy within the fields of counselling and psychotherapy. The original question asked: is it appropriate to consider integrating email communication in to stammering therapy? With specific rationale for using email with individual clients, and with full client consent, it is argued that email can be integrated in to treatment in the context of the relevant state or country licensing laws. Future research is needed to understand the experience of using email therapeutically from a client perspective and to investigate the efficacy of therapeutic

email components in treatment. This will ensure that the use of technology-based communication enhances, and does not detract from, a quality therapeutic service. 


\section{Acknowledgments}

I wish to thank my clients for giving permission to incorporate their email messages into this paper and Professor Catherine Mackenzie for review and guidance regarding the manuscript.

\section{References}

1. Royal College of Speech and Language Therapists. RCSLT Clinical Guidelines. London: Speechmark, 2005

2. Mashima PA, Doarn CR. Overview of telehealth activities in speech-language pathology. Telemed J E Health 2008;14:1101-17

3. Reynolds AL, Vick JL, Haak NJ. Telehealth applications in speech-language pathology: a modified narrative review. J Telemed Telecare 2009;15:310-6

4. Ward E, Crombie J, Trickey M, Hill A, Theodoros D, Russell T. Assessment of communication and swallowing post-laryngectomy: a telerehabilitation trial. J Telemed Telecare 2009;15:232-7 
5. Mashima PA, Birkmire-Peters DP, Syms MJ, Holtel MR, Burgess LPA, Peters LJ. Telehealth: voice therapy using telecommunications technology. Am J Speech Lang Pathol 2003;12:432-439

6. Kully D. Telehealth in speech pathology: applications to the treatment of stuttering. J Telemed Telecare 2000;6:39-41

7.Carey B, O’Brian S, Onslow M, Block S, Jones M, Packman A. Randomized controlled non-inferiority trial of a telehealth treatment for chronic stuttering: the Camperdown Program. Int J Lang Comm Dis 2010;45:108-120

8. Manning W, Hodak M, Plexico L. Letters from Sarajevo. Available from: URL: http://www.mnsu.edu.comdis.isad8/papers/manning8.html. (last accessed 01-09-09)

9. Harrrison E. Technology in stuttering treatment for children and adults. Presented at the $6^{\text {th }}$ World Congress on Fluency Disorders; 2009 Aug 5-8, Rio de Janeiro, Brazil

10. Wright L \& Ayre A. Wright \& Ayre Stuttering Self Profile. Bicester:Winslow;2000 
11. James SE, Brumfitt SM, Cudd PA. Communicating by telephone: views of a group of people with stuttering impairment. J Fluency Disord 1999;24:299-317

12. Hjelm NM. Benefits and drawbacks of telemedicine. J Telemed Telecare 2005;11:60-70

13. Murphy LJ, Mitchell DL. When writing helps to heal: e-mail as therapy. Br J Guid Counc 1998;26:21-32

14. Rochlen AB, Zack JS, Speyer C. Online therapy: review of relevant definitions, debates, and current empirical support. J Clin Psychol 2004;60:269-283

15. O'Brian S, Packman A, Onslow M. Telehealth delivery of the Camperdown program for adults who stutter: a phase I trial. J Speech Lang Hear Res 2008;51:184-195

16. Bolton G. The therapeutic potential of creative writing. Writing myself. London: Jessica Kingsley Publishers;1999 
17. Brice A. Therapeutic support using e-mail: a case study. Counselling $2000 ; 11 ; 100-101$

18. Leibert T, Archer Jr. J, Munson J, York G. An exploratory study of client perceptions of internet counseling and the therapeutic alliance. J Ment Health Counsel 2006;28:69-83

19. Sweet $\mathrm{H}$. Finding the person behind the persona: engaging men as a female therapist. In: Englar-Calson M \& Stevens MA, editors. In the room with men: a casebook of therapeutic change. Washington DC: American Psychological Associaton;2006. p. 69-90.

20. Skårderud F. Sh@me in cyberspace. Relationships without faces: the e-media and eating disorders. Eur Eat Disord Rev 2003;11:155-169

21. Robinson PH, Serfat MA. The use of e-mail in the identification of bulimia nervosa and its treatment. Eur Eat Disord Rev 2001;9:182-193 
22. Cartwright M, Gibbon P, McDermott BM, Bor W. The use of email in a child and adolescent mental health service: are staff ready? J Telemed Telecare 2005;11:199204

23. Neville RG, Marsden W, McCowan C, Pagliari C, Mullen H, Fannin A. Email consultations in general practice. Inform Prim Care 2004;12:207-214

24. Ling Leong S, Gingrich D, Lewis PR, Mauger DT, George JH. Enhancing doctorpatient communication using email: a pilot study. J Am Board Fam Pract 2005;18:180-188

25. Denton DR. Ethical and legal issues related to telepractice. Semin Speech Lan. $2003 ; 24: 313-322$

26. Stanberry B. Legal and ethical aspects of telemedicine. J Telemed Telecare 2006;12:166-175

27. Robson D, Robson M. Intimacy and computer communication. Br J Guid Counc. 1998;26:33-40 
28. Reynolds Jr DJ, Stiles WB, Grohol JM. An investigation of session impact and alliance in internet based psychotherapy: preliminary results. Counsel Psychother Res 2006;6:164-168

29. Goss S, Anthony K. Developments in the use of technology in counselling and psychotherapy. Br J Guid Counc. 2009;37:223-230

30. Buck S. Nine human factors contributing to the user acceptance of telemedicine applications: a cognitive-emotional approach. J Telemed Telecare 2009;15:55-58

31. Bothe AK. Evidence-based treatment of stuttering: V. The art of clinical practice and the future of clinical research. J Fluency Disord 2003;28:247-258 